Gefässchirurgie 2012 • 17:582-584

DOI 10.1007/s00772-012-1064-x

Online publiziert: 26. September 2012

(c) Deutsche Gesellschaft für Gefäßchirurgie und Gefäßmedizin - Gesellschaft für operative, endovaskuläre und präventive Gefässmedizin e.V. Published by Springer-Verlag all rights reserved 2012

\section{Gesellschaftszugehörigkeit}

Joachim Berkefeld: Deutsche Gesellschaft für Neuroradiologie (DGNR)

Arnd Dörfler: Deutsche Gesellschaft für Neuroradiologie (DGNR)

\subsubsection{Empfehlungen}

Siehe $\bullet$ Tab. 1.

\subsubsection{Literatur (Leitlinien, Reviews, Studien), Bewertung der Evidenz und offene Fragen}

\section{Leitliniensynopse und systematische}

Reviews: In der Mehrzahl der aktuellen LL finden sich keine Details zu technischen Fragen der endovaskulären Behandlung. In der ESVS-LL [1] (2009, - Tab. 17 in der Onlineversion unter http://www.awmf.org) werden Carotisschleusen, selbstexpandierende Stents und die Verwendung eines Protektionssystems als Standard angegeben. In der AHA-Leitlinie [2] werden Protektionssysteme als nützlich angesehen (• Tab. 11 in der Onlineversion unter http://www. awmf.org). In der aktuellen LL der ESC [3] wird eine schwache Empfehlung gegeben, bei CAS ein Protektionssystem anzuwenden (Class IIb, LoE B, - Tab. 13

J. Berkefeld ${ }^{1} \cdot$ A. Dörfler ${ }^{2}$

${ }^{1}$ Zentrum der Neurologie und Neurochirurgie, Hirngefäßzentrum, Klinikum und

Fachbereich Medizin, Johann Wolfgang Goethe-Universität Frankfurt

${ }^{2}$ Neuroradiologische Abteilung, Universitätsklinikum Erlangen

\title{
S3-Leitlinie Extracranielle Carotisstenose
}

\author{
Kapitel 8.13: Welche Materialien \\ (Katheter, Stents, Protektionssysteme) \\ sind bei CAS zu bevorzugen?
}

in der Onlineversion unter http://www. awmf.org).

\section{Bewertung der Evidenz und offene Fragen:}

- Stent-Typ: Es gibt keine RCTs zum Vergleich verschiedener CarotisstentTypen. Mit Veröffentlichung der ersten großen Fallserien Ende der 1990er Jahre ergaben sich Hinweise darauf, dass ballonexpandierbare, durch Druck von außen deformierbare Stents für die oberflächlich gelegene Carotisbifurkation ungeeignet sind [4], sodass sich selbstexpandierende Stents als Standard durchgesetzt haben. Daher werden an der Carotisbifurkation nahezu ausschließlich selbstexpandierende Stents verwendet. Bei hochzervikalen und intracraniellen ACI-Stenosen sowie am ACCAbgang sind auch ballonexpandierbare Stents möglich und aufgrund ihrer schnellen und exakten Platzierbarkeit vielfach zu bevorzugen. Nach einer Subgruppenanalyse aus der SPACEI-Studie und einigen Fallserien waren Komplikationen bei Verwendung von Stents mit geschlossenem Zell-Design weniger häufig als bei der Verwendung von offenzelligen Nickel-TitanStents [5, 6]. Andere Studien konnten diesen Unterschied nicht bestätigen [7]. In großen Registern und Zulas- sungsstudien sind die Komplikationsraten mit verschiedenen CarotisstentTypen ähnlich $[8,9,10]$. Das StentDesign hat $u$. U. auch einen Einfluss auf die Restenoserate, die bei der Verwendung konischer Nickel-TitanStents geringer sein soll als bei Stents mit einheitlichem Durchmesser [11].

- Welchen Stellenwert haben Neuroprotektionssysteme bei der interventionellen Therapie? Auch zur Frage, ob Protektionssysteme die Sicherheit von CAS-Eingriffen erhöhen, existieren keine Daten aus explizit dazu durchgeführten RCTs. Registerdaten und Metaanalysen aus der Zeit vor Veröffentlichung der großen RCTs ergaben Hinweise auf eine mögliche Überlegenheit der Stentimplantation unter Verwendung von Protektionssystemen [12]. Eine Subgruppenanalyse [6] der SPACE-1Studie konnte keinen signifikanten Vorteil der Anwendung von Embolieschutzsystemen gegenüber einer Stentimplantation ohne Protektion nachweisen. Ebenso gibt es Fallserien mit niedrigen Komplikationsraten und wenigen DWI-Läsionen bei CAS

Die Leitlinie Extracranielle Carotisstenose; Diagnostik, Therapie und Nachsorge wird bei der AWMF unter der Register-Nummer 004-028 geführt. 
Tab. 1 Welche Materialien (Katheter, Stents, Protektionssysteme) sind bei CAS zu

bevorzugen?

\begin{tabular}{|c|c|c|c|}
\hline Nr. & Text der Empfehlung & Grad $^{a}$ & $\operatorname{LoE}^{b}$ \\
\hline A & $\begin{array}{l}\text { Für die Carotisstentimplantation sollen in jedem Fall selbstexpandie- } \\
\text { rende, für diese Indikation zugelassene Stents verwendet werden }\end{array}$ & 个个 & 3 \\
\hline \multicolumn{4}{|c|}{ 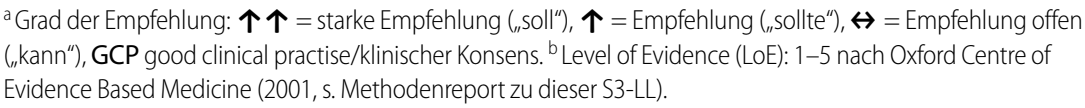 } \\
\hline
\end{tabular}

ohne Protektion [6, 13]. Die CAS-Patienten in der EVA-3S und ICSS-Studie hatten trotz weit gehender Verwendung von Protektionssystemen relativ hohe Komplikationsraten [14, 15]. Die Daten aus DWI-Studien belegen, dass mikroembolische Ischämien trotz Protektion vorkommen, in der ICSS-Substudie sogar signifikant häufiger als nach CEA [16]. In den für den Carotisstent relativ günstigen RCTs SAPPHIRE und CREST war ein Protektionssystem vorgeschrieben mit der Besonderheit, dass jeweils nur ein Filtertyp verwendet werden durfte. Einen signifikanten Unterschied der Rate zerebraler Komplikationen im Vergleich zu Studien ohne Protektionssystem (SPACE) besteht nicht. Daten aus Zulassungsstudien, Registern und nicht randomisierten Vergleichsstudien zeigen für die verschiedenen Protektionssysteme ähnliche Ergebnisse ohne Hinweise auf eine Überlegenheit einer bestimmten Technik (BEACH [9], CARAT, SAPPHIRE [10], ARMOUR [17]). Mit proximalen Protektionssystemen gewonnene Registerdaten und konzeptionelle Überlegungen deuten darauf hin, dass das sog. „endovascular clamping “ mit Ballonokklusion der ACC und ACE zu niedrigeren Komplikationsraten führen könnte [17, 18]. Diese Hypothese müsste allerdings durch weitere Studien überprüft werden.

- Zugangssysteme: Mit Einführung spezieller langer Schleusen und Führungskatheter ist der interventionelle Zugang zur A. carotis einfacher und theoretisch auch komplikationsärmer möglich. Diese für CAS-Prozeduren wichtigen Materialien sind in Studien nur unzureichend evaluiert worden.
Bewertung der Evidenz und offene

Fragen: Bisher ist es nicht gelungen, valide RCTs mit ausreichenden Fallzahlen zum Vergleich verschiedener CASTechniken durchzuführen. Dementsprechend sind jenseits von praktischen Empfehlungen keine klaren Aussagen darüber möglich, welche Technik am besten und risikoärmsten ist. Daten aus Subgruppenanalysen und offenen Studien können allenfalls zur Hypothesenbildung beitragen, erlauben aber keine belastbaren Empfehlungen. Im Rahmen der klinischen Praxis haben sich selbstexpandierende Stents verbindlich durchgesetzt. Die Frage, ob die Komplikationsrate oder die Langzeithängen, lässt sich nicht zuverlässig beantworten. Angesichts fehlender Daten mit hohem Evidenzniveau kann die Verwendung von Protektionssystemen nicht uneingeschränkt empfohlen werden. Protektionssysteme können die Sicherheit des Eingriffs, insbesondere den Schutz vor Makroembolien, bei entsprechender Erfahrung, erhöhen $[1,19]$ ( Tab. 10 und $\bullet$ Tab. 17 in der Onlineversion unter http://www.awmf.org). Die Verwendung erhöht die Komplexität des Eingriffs und erfordert ein entsprechendes Training und Erfahrung.

\section{Korrespondenzadressen}

\section{Prof. Dr. J. Berkefeld}

Zentrum der Neurologie und Neurochirurgie, Hirngefäßzentrum, Klinikum und Fachbereich Medizin, Johann Wolfgang Goethe-Universität Frankfurt Theodor-Stern-Kai 7, 60590 Frankfurt am Main berkefeld@em.uni-frankfurt.de

\section{Prof. Dr. A. Dörfler}

Neuroradiologische Abteilung, Universitätsklinikum Erlangen Schwabachanlage 6, 91054 Erlangen arnd.doerfler@uk-erlangen.de ergebnisse vom Stent-Typ und Design ab-
Interessenkonflikt. Die korrespondierenden Autoren geben an, dass kein Interessenkonflikt besteht.

\section{Literatur}

1. Liapis CD, Bell PR, Mikhailidis D et al (2009) ESVS guidelines. Invasive treatment for carotid stenosis: indications, techniques. Eur JVasc Endovasc Surg 37:1-19

2. Goldstein LB, Bushnell CD, Adams RJ et al (2011) Guidelines for the primary prevention of stroke: a guideline for healthcare professionals from the American Heart Association/American Stroke Association. Stroke 42:517-584

3. Tendera M, Aboyans V, Bartelink ML et al (2011) ESC Guidelines on the diagnosis and treatment of peripheral artery diseases: document covering atherosclerotic disease of extracranial carotid and vertebral, mesenteric, renal, upper and lower extremity arteries *The Task Force on the Diagnosis and Treatment of Peripheral Artery Diseases of the European Society of Cardiology (ESC). Eur Heart J 32:2851-2906

4. Yadav JS, Roubin GS, lyer $S$ et al (1997) Elective stenting of the extracranial carotid arteries. Circulation 95:376-381

5. Hart JP, Bosiers M, Deloose K et al (2010) Impact of stent design on the outcome of intervention for carotid bifurcation stenosis. J Cardiovasc Surg (Torino) 51:799-806

6. Jansen O, Fiehler J, Hartmann M, Bruckmann H (2009) Protection or nonprotection in carotid stent angioplasty: the influence of interventional techniques on outcome data from the SPACE Trial. Stroke 40:841-846

7. Jim J, Rubin BG, Landis GS et al (2011) Society for vascular surgery vascular registry evaluation of stent cell design on carotid artery stenting outcomes. J Vasc Surg 54:71-79

8. Gray WA, Yadav JS, Verta P et al (2007) The CAPTURE registry: results of carotid stenting with embolic protection in the post approval setting. Catheter Cardiovasc Interv 69:341-348

9. White CJ, lyer SS, Hopkins LN et al (2006) Carotid stenting with distal protection in high surgical risk patients: The BEACH trial 30 day results. Catheter Cardiovasc Interv 67:503-512

10. Massop D, Dave R, Metzger C et al (2009) Stenting and angioplasty with protection in patients at high-risk for endarterectomy: SAPPHIRE Worldwide Registry first 2,001 patients. Catheter Cardiovasc Interv 73:129-136

11. Brown KE, Usman A, Kibbe MR et al (2009) Carotid stenting using tapered and nontapered stents: associated neurological complications and restenosis rates. Ann Vasc Surg 23:439-445

12. Kastrup A, Nagele T, Groschel K et al (2006) Incidence of new brain lesions after carotid stenting with and without cerebral protection. Stroke 37:2312-2316

13. Tietke MW, Kerby T, Alfke K et al (2010) Complication rate in unprotected carotid artery stenting with closed-cell stents. Neuroradiology 52:611-618

14. Mas JL, Chatellier G, Beyssen B et al (2006) Endarterectomy versus stenting in patients with symptomatic severe carotid stenosis. N Engl J Med 355:1660-1671

15. Ederle J, Dobson J, Featherstone RL et al (2010) Carotid artery stenting compared with endarterectomy in patients with symptomatic carotid stenosis (International Carotid Stenting Study): an interim analysis of a randomised controlled trial. Lancet 375:985-997 


\section{Leitlinien}

16. Bonati LH, Jongen LM, Haller S et al (2010) New ischaemic brain lesions on MRI after stenting or endarterectomy for symptomatic carotid stenosis: a substudy of the International Carotid Stenting Study (ICSS). Lancet Neurol 9:353-362

17. Ansel GM, Hopkins LN, Jaff MR et al (2010) Safety and effectiveness of the INVATEC MOMA proximal cerebral protection device during carotid artery stenting: results from the ARMOUR pivotal trial. Catheter Cardiovasc Interv 76:1-8

18. Micari A, Stabile E, Cremonesi A et al (2010) Carotid artery stenting in octogenarians using a proximal endovascular occlusion cerebral protection device: a multicenter registry. Catheter Cardiovasc Interv 76:9-15
19. Brott TG, Halperin JL, Abbara S et al (2011) 2011 ASA/ACCF/AHA/AANN/AANS/ACR/ASNR/CNS/ SAIP/SCAI/SIR/SNIS/SVM/SVS Guideline on the Management of patients with extracranial carotid and vertebral artery disease: executive summary A Report of the American College of Cardiology Foundation/American Heart Association Task Force on Practice Guidelines, and the American Stroke Association, American Association of Neuroscience Nurses, American Association of Neurological Surgeons, American College of Radiology, American Society of Neuroradiology, Congress of Neurological Surgeons, Society of Atherosclerosis Imaging and Prevention, Society for Cardiovascular Angiography and Interventions, Society of Interventional Radiology, Society of Neurolnterventional Surgery, Society for Vascular Medicine, and Society for Vascular Surgery Developed in Collaboration With the American Academy of Neurology and Society of Cardiovascular Computed Tomography. J Am Coll Cardiol 57:1002-1044

\section{CME.springer.de wird zur e.Akademie}

Die e.Akademie von Springer Medizin ist die Weiterentwicklung von CME.springer.de und bietet Ihnen ein noch umfassenderes und moderneres Fortbildungsangebot: Mehr als 1500 Fortbildungsmodule, neue e.Learningformate und multimediale Elemente machen Ihre Fortbildung und das Sammeln von CME-Punkten noch flexibler und komfortabler.

e.CME: Als Zeitschriftenabonnent stehen Ihnen in der e.Akademie nach wie vor alle zertifizierten Fortbildungsbeiträge Ihrer Zeitschrift als e.CME zur Verfügung. Darüber hinaus haben Sie künftig die Möglichkeit, Beiträge Ihrer Zeitschrift, deren Zertifizierungszeitraum abgelaufen ist, weiterhin für Ihre Fortbildung und persönlichen Wissenscheck zu nutzen.

- Der direkte Weg zur e.Akademie unter springermedizin.de/eAkademie
Nutzer von e.Med, dem Springer Medizin Online-Paket, profitieren vom vollen Leistungsumfang der e.Akademie: Mehr als 1500 e.CMEs aus den wichtigsten medizinischen Fachgebieten werden ergänzt durch die neuen e.Learningformate e.Tutorial und e.Tutorial plus.

Das e.Tutorial ist speziell für die Online-Fortbildung konzipiert und didaktisch optimiert. Klar gegliederte Lernabschnitte, besondere Hervorhebung von Merksätzen, zoomfähige Abbildungen und Tabellen sowie verlinkte Literatur erleichtern das Lernen und den Erwerb von CME-Punkten.

Das e.Tutorial.plus bietet multimedialen Zusatznutzen in Form von Audio- und Videobeiträgen, 3D-Animationen, Experteninterviews und weiterführende Informationen. CME-Fragen und Multiple-Choice-Fragen innerhalb der einzelnen Lernabschnitte ermöglichen die Lernerfolgskontrolle.

- Weitere Informationen zum e.Med-Komplettpaket und Gratis-Testangebot unter springermedizin.de/eMed 